\title{
déformation d'un échantillon de sable sur chemins de contrainte isotrope et triaxiaux de révolution: influence de l'histoire
}

\author{
par \\ J. Lanier \\ Maître assistant \\ Institut de Mécanique de Grenoble
}

\section{Introduction}

Lorsque l'on recherche la formulation de la loi de comportement d'un matériau simple, on admet le principe du déterminisme selon lequel la réponse actuelle du matériau est une fonctionnelle de l'histoire subie par celui-ci. Nous proposons dans cet article une étude expérimentale de la réponse en déformation d'un échantillon de sable sec sur chemin de contrainte isotrope, cet échantillon ayant subi une sollicitation triaxiale de révolution à pression latérale constante auparavant.

Quelques auteurs ont déjá étudié ce problème : Biarez (1962). Elshoby et Andrawes (1973), Yamada et Ishimara (1979). Ils ont montré que la réponse du matériau était anisotrope. Nous complétons ici leur étude en montrant comment cette anisotropie dépend de la déformation triaxiale qui a précédé et comment les essais triaxiaux de compression et d'extension donnent lieu à une anisotropie différente.

Les réșultats présentés ont été obtenus sur la presse véritablement triaxiale de Grenoble, Lanier (1976).

\section{Description de la presse et hypothèses}

2.1. La presse comporte 6 plaques formant un parallélépipède rectangle déformable dont les dimensions d'arêtes $\mathrm{Xi}$ sont variables

Le mouvement d'avance ou de recul de 2 faces opposées est réalisé par 2 moteurs électriques synchronisés. La vitesse maximale est de $5 \mathrm{~mm} / \mathrm{mn}$. Trois capteurs à induction mesurent les dimensions d'arête, trois capteurs "noyés" dans les plaques mesurent les contraintes normales.
2.2 Pour interpréter ces mesures nous faisons deux hypothèses

1) L'état de déformation est homogène pour un échantillon homogène.

2) Les dimensions principales des tenseurs de contraintes et de déformations coïncident pour toutes les cinématiques imposées par la presse.

En conséquence, les trois contraintes normales mesurées sont les contraintes principales.

La première hypothèse est difficile à contrôler. Aussi pour être dans les meilleures conditions d'essais possibles, un film de graisse aux silicones est appliqué sur les plaques afin de diminuer le trottement avec l'enveloppe de caoutchouc (épaisseur $0.5 \mathrm{~mm}$ ) qui contient l'échantillon.

La deuxième hypothèse concerne la loi de comportement du matériau.

2.3 La régulation, entièrement électronique, permet d'imposer indépendamment dans chacune des trois directions:

- une vitesse de plaque $v_{i}(t)$;

- une contrainte $\sigma_{i}(t)$;

- une dimension $X_{i}(t)$.

Ces consignes analogiques peuvent être engendrées de trois façons :

- par potentiomètre (consigne constante par morceaux):

- par un calculateur analogique réalisant les 2 fonctions.
(1) $S_{1}=c-\left(E_{1}+E_{2}\right)$
(2) $\left\{\begin{array}{l}S_{1}=a E_{1}+b \\ S_{2}=c E_{2}+d\end{array}\right.$ 
où $E_{1}$ et $S$ sont les signaux d'entrée et de sortie - par trois convertisseurs digitaux-analogiques incorporés dans une centrale à micro-processeur.

\section{Présentation des essais}

\subsection{Préparation et mise en place de l'échantillon}

L'échantillon est préparé dans une capote, en latex, cubique de dimension $100 \times 100 \times 100 \mathrm{~mm}^{3}$, à l'intérieur d'un moule de même dimension. Le sable est déversé et damé par couche d'environ $1 \mathrm{~cm}$ d'épaisseur. La face de la capote qui sert au remplissage est fermée avec une rustine, en latex également, de $90 \mathrm{~mm}$ dę diamétre. Un léger vide intérieur permet le démoulage et le transport de l'échantillon sur la presse sans le remanier.

\subsection{Régulations utilisées}

Au cours d'un essai deux types de régulation sont utilisées.

3.2.1 Chemin isotrope en contrainte: après avoir approché les plaques au contact de l'échantillon $\sigma_{X}=v_{y}=v_{Z} \# 0$ on impose une vitesse de compression dans une direction, $\mathrm{Z}$ par exemple, et l'on utilise la régulation analogique

$\sigma_{X}=v_{z}$

$\sigma_{x}=u_{z}$

On réalise ainsi une charge isotrope. Si l'on inverse le sens de la vitesse on obtiendra une décharge isotrope.

Ce type de régulation peut être utilisé à partir d'un état de contrainte isotrope quelconque

3.2.2 Chemins triaxiaux de révolution : à partír d'un état de contrainte isotrope, $\sigma_{X}=\sigma_{Y}=\sigma_{Z}=0,3 \mathrm{MPa}$ par exemple on peut simuler l'essai triaxial classique en imposant 2 contraintes constantes égales à $0,3 \mathrm{MPa}$ (régulation par potentiomètre) et une vitesse de compression ou d'extension dans la direction choisie comme axe de révolution pour l'état de contrainte. Nous noterons dans la suite $\operatorname{Tr} i \Phi_{1}^{c}$ (ou $\operatorname{Tr} \Phi_{1}^{E}$ ) les essais triaxiaux de révolution en compression (ou en extension) sur la direction $\mathrm{i}(\mathrm{i}=\mathrm{X}, \mathrm{Y}$ ou $\mathrm{Z})$.

\subsection{Représentation du chemin de contrainte}

Si nous faisons successivement un essai triaxial et un essai isotrope le chemin de contrainte suivi peut être représentè dans le plan $(p, q)$ où $p=1 / 3$ tr u (pression moyenne) et $q=[\operatorname{tr}(v-p \delta)(v-p \delta)]^{1 / 2}$ (intensité $d u$ déviateur).

Rappelons que pour les états de contraintes de révolution, pour lesquels les contraintes principales sont ${ } r_{1}, \sigma_{2}=w_{3}$ on a :

$\mathrm{p} .=\left(\sigma_{1}+2 \sigma_{2}\right) / 3$

$q=\sqrt{2 / 3}\left|\sigma_{1}-s_{2}\right|$

Les chemins isotropes sont représentés par $q=0$ et les chemins triaxiaux de révolution à $\sigma_{2}$ constant par $q=\sqrt{6}\left|p-r_{2}\right|$

\subsection{Essais présentés}

Nous présentons les résultats de 2 essais, notés Sable 15 et Sable 17. On peut suivre sur les figures 1 et 8 l'évolution des contraintes et du volume en fonction d'un paramètre $s$ défini par $s=\int_{0}^{1}\left[\operatorname{trd} f^{2}\right]^{1 / 2}, \mathrm{Ce}$ parametre, toujours croissant avec la déformation, joue le rôle d'un pseudo-temps. On observe ainsi aisément la succession d'essais isotropes pour lesquels les contraintes varient de 0,3 à $2,0 \mathrm{MPa}$ et les essais triaxiaux de révolution pour lesquels 2 contraintes restent constantes égales à $0,3 \mathrm{MPa}$. Pour l'essai Sable 15 nous avons réalisé successivement (figures 1 et 2) avec le même échantillon les 13 séquences suivantes :

1) Essai isotrope initial $\sigma_{X}=\sigma_{Y}=\sigma_{Z}:$ charge de 0,3 à 2,0 MPa et décharge jusqu'à $0,3 \mathrm{MPa}$.

2) Essai triaxial de révolution par rapport à la direction Z. $\sigma_{X}=\sigma_{Y}=0,3 \mathrm{MPa}$ compression dans la direction $Z$ jusqu'à $\gamma_{Z}^{\circ}=5 \%$ et décharge jusqu'à l'état isotrope $\sigma_{x}=\sigma_{y}=\sigma_{z}=0.3 \mathrm{MPa}$.

3) Essai isotrope (cf. 1)

4) Essai triaxial (cf. 2) jusqu'à $\kappa_{z}^{0}=10 \%$ et décharge.

5) Essai isotrope (cf. 1)

6) Essai triaxial (cf. 2) jusqu'à $\varepsilon_{Z}^{0}=20 \%$ et décharge.

7) Essai isotrope (cf. 1)

8) Essai triaxial (cf. 2) jusqu'à $y_{z}^{0}=35 \%$ et décharge.

9) Essai isotrope (ct. 1)

10) Essai triaxial de révolution par rapport à la direction $X: v_{Y}=v_{Z}=0,3 \mathrm{MPa}$; compression dans la direction $X$ jusqu'à $\varepsilon_{X}=15 \%$ et décharge.

11) Essai isotrope (cf. 1).

12) Essai triaxial de révolution par rapport à la direction $Y: \sigma_{X}=\sigma_{Z}=0.3 \mathrm{MPa}$; compression dans la direction $Y$ jusqu'à $F_{Y}=15 \%$ de décharge:

13) Essai isotrope (cf. 1)

De même pour l'essai Sable 17 le chemin suivi se décompose en 6 séquences (fig. 8 ).

1) Essai triaxial de révolution par rapport à l'axe $Z$ $\tau_{X}=v_{y}=0,3 \mathrm{MPa}$; extension dans la direction $Z$ jusqu'à $\xi_{z}=-10 \%$ et recharge jusqu'à l'état isotrope $\omega_{x}=w_{Y}=\omega_{z}=0.3 \mathrm{MPa}$.

2) Essai isotrope $\omega_{x}=\sigma_{y}=\sigma_{z}:$ charge de 0,3 à 2,0 MPa et décharge jusqu'à $0.3 \mathrm{MPa}$.

3) Essai triaxial de révolution par rapport à l'axe $Y$, $w_{x}=w_{Z}=0,3 \mathrm{MPa}$; extension dans la direction $Y$ jusqu'à $\mathrm{F}_{\gamma}=-10 \%$ et recharge.

4) Essai isotrope.

5) Essai triaxial de révolution par rapport à l'axe $Y$, $\omega_{X}=\sigma_{Z}=0.3 \mathrm{MPa}$; compression jusqu'à $F_{Y}=$ $+20 \%$ et décharge.

6) Essai isotrope : charge de 0,3 à 1,0 $\mathrm{MPa}$; dècharge jusqu'à $0,3 \mathrm{MPa}$; recharge jusqu'à $2,0 \mathrm{MPa}$ et décharge jusqu'à $0,3 \mathrm{MPa}$.

Les déformations utilisées sont les déformations logarithmiques $\varepsilon_{1}=-\log X_{1} / X_{1}^{\circ}$ positives en compression. négatives en extension. La configuration de référence, $X_{i}^{0}$, est la configuration du début de chaque séquence sauf pour l'essai Sable 15 où $q_{z}^{\circ}$ représente la déformation de la direction $Z$ à partir de la configuration initiale.

\section{Anisotropie créée par les essais triaxiaux de compression (Sable 15)}

\subsection{Cycle isotrope après les Tri $\Phi_{z}^{c}$}

L'essai Sable 15 comporte un essai isotrope initial puis quatre cycles de charge-décharge triaxiales suivi chacun par un essai isotrope (cf. figure 1). Soit $\varepsilon_{2}^{0}$ la déformation dans la direction $\mathrm{Z}$ mesurée à partir de la configuration initiale. La figure 2 représente $\sigma_{z}\left(\varepsilon_{z}^{0}\right)$. Les cycles isotropes (1) à (5) pour lesquels $\sigma_{z}$ varie entre 0.3 et $2,0 \mathrm{MPa}$ ne sont pas représentés. La figure 3 représente les déformations $\varepsilon_{x}, \varepsilon_{y}, \varepsilon_{z}$ en fonction de la 

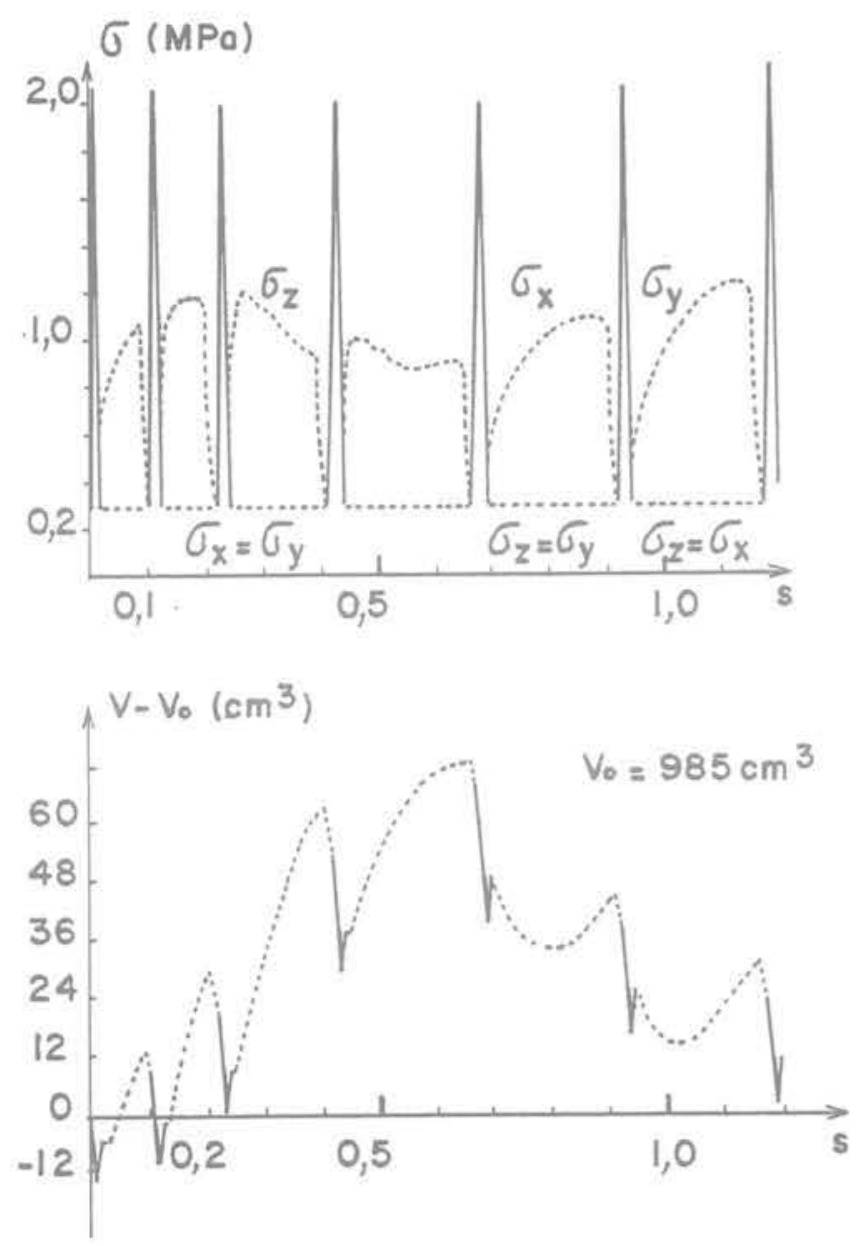

Fig. 1 Evolution des contraintes et du volume (sable 15) essais triaxiaux de révolution essais isotropes

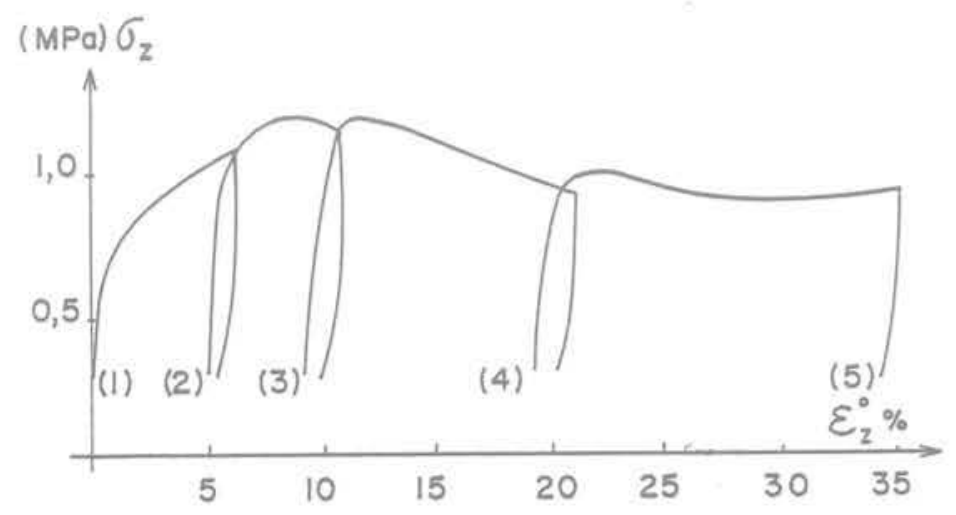

Fig. 2 Essai triaxial de révolution: $\sigma_{z}(\epsilon)$ Pression latérale : 0,3 MPa (sable 15) (les essais isotropes (1) à (5) ne sont pas représentés) pression moyenne pour les 5 premiers cycles isotro-

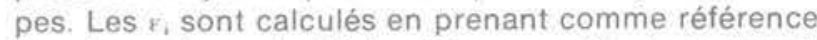
les dimensions de l'échantillon au début de l'essai isotrope correspondant.

Si l'échantillon était isotrope les trois \& devraient être égaux, or on constate que ce n'est pas le cas.

L'essai initial présente une légère anisotropie que l'on peut attribuer au mode de préparation et à la mise en place de l'échantillon sur la presse. Par contre les quatre essais suivants indiquent nettement la création d'une orthotropie de révolution autour de la direction $Z$. En effet, $r_{x}$ et $r_{Y}$ sont peu différents, et nettement supérieurs à $\mathrm{F}_{Z}$

Autrement dit, la déformation dans l'essai triaxial de révolution a entrainé une modification de la structure de l'échantillon: les directions latérales se sont "amollies", la direction de compression s'est "durcie".

Le fait que $d_{z_{z}} / d p$ soit nettement négatif pour les essais 3-4-5 doit être compris comme un "effet de Poisson $n$ : la dilatation provoquée sur la direction Z par l'augmentation des contraintes latérales est prépondérante vis-à-vis de la contraction due à l'augmentation de $i r_{\mathrm{z}}$.

En effet, une loi de type incrémentale linéaire et orthotrope de révolution entre dir et $d r$ s'écrit avec des notations classiques pour ce type de sollicitations (Darve et al. (1978)) :

$\mathrm{d} \varepsilon=\mathrm{Ad} i s$

avec :

$A=\left|\begin{array}{rrr}\frac{1}{E_{x}} & -\frac{v_{x}^{x}}{E_{x}} & -\frac{v_{z}^{x}}{E_{z}} \\ -\frac{v_{x}^{y}}{E_{x}} & \frac{1}{E_{x}} & -\frac{v_{z}^{x}}{E_{z}} \\ -\frac{v_{x}^{z}}{E_{x}} & -\frac{v_{x}^{z}}{E_{x}} & \frac{1}{E_{z}}\end{array}\right|$

où $E_{1}, v_{\text {l }}$ sont des pseudo-modules d'Young et de Poisson.

$d c=\left(d v_{x}, d \tau_{\gamma}, d v_{z}\right)$ et $d y=\left(d z_{x}, d f_{\gamma}, d s_{z}\right)$.

Pour $d r=(d p, d p, d p)$ on en déduit :

$d r_{x}=d r_{y}=\left[\left(1-v_{x}^{x}-v_{z}^{x} \cdot E_{x} / E_{z}\right) / E_{x}\right] \cdot d p$

$d z_{z}=\left[\left(1-21, z \cdot E_{z} / E_{x}\right) / E_{z}\right] d \rho$

$d_{i z} / d p$ sera nègatif si $2 v_{x}^{z}>E_{x} / E_{z}$

4.2 Évolution de l'anisotropie avec la déformation triaxiale de révolution

On note $r_{Z}^{0}$ la déformation au début de l'essai isotrope dans la direction $\mathrm{Z}$, mesurée à partir de la configuration initiale.

Pour caractériser l'orthotropie nous posons:

$\Delta(p)=\frac{p_{x}+p_{x}}{2}-r_{z}$

où $f_{X}, F_{Y}, \varepsilon_{Z}$, sont les déformations au cours de l'essai Isotrope.

Pour un échantillon isotrope $f_{X}=f_{Y}=r_{z}$ et $\Delta=0 \mathrm{Vp}$. Nous avons représenté figure 4 l'évolution de

$\Delta(\mathrm{p}=2 \mathrm{MPa})$

en fonction de $r_{z}^{\circ}$. On constate que $\Delta$ augmente avec $\varepsilon_{z}^{\circ}$ puis atteint une valeur limite pour $₹_{Z}^{\circ} \# 20 \%$ ce qui indique que "l'état " d'anisotropie reste stationnaire pour $\varepsilon_{Z}^{0}>20 \%$. Si l'on se reporte à la courbe $\sigma_{z}\left(\varepsilon_{z}^{\circ}\right)$ 
(1) $\varepsilon_{z}^{0}=0 \%$

(MPa) $p$

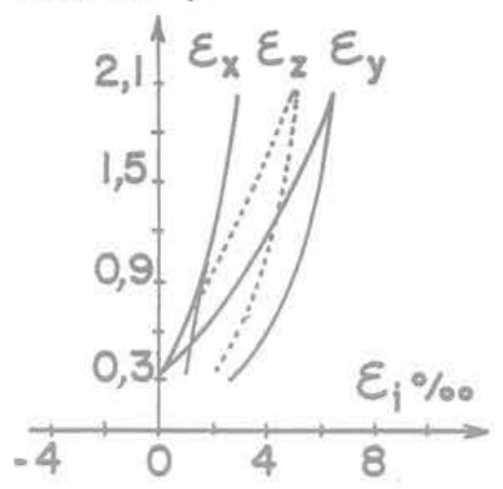

(3) $\varepsilon_{z}^{0}=8 \%$

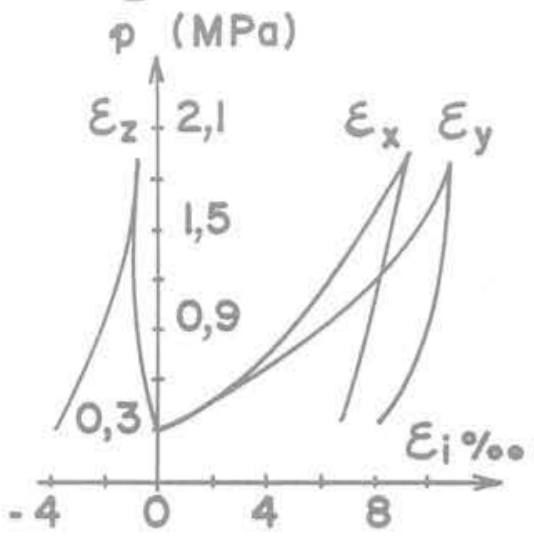

(2) $\varepsilon_{z}^{0}=4 \%$

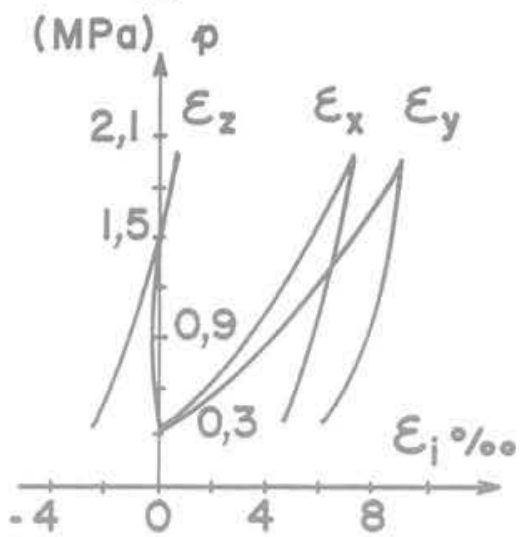

(4) $\varepsilon_{z=16 \%}^{0}$

(5) $\varepsilon_{z}^{0}=27 \%$

$\mathrm{p}(\mathrm{MPa})$

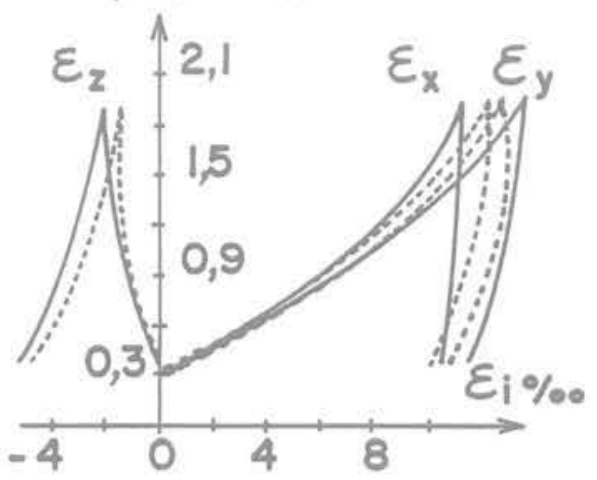

Fig. 3 Déformations $\epsilon_{x} \epsilon_{y} \epsilon_{z}$ au cours des essais isotropes: $\sigma_{x}=\sigma_{y}=\sigma_{z}=p$ (sable 15) 1 : essai initial ; $2,3,4,5$ : après Tri $\varnothing$

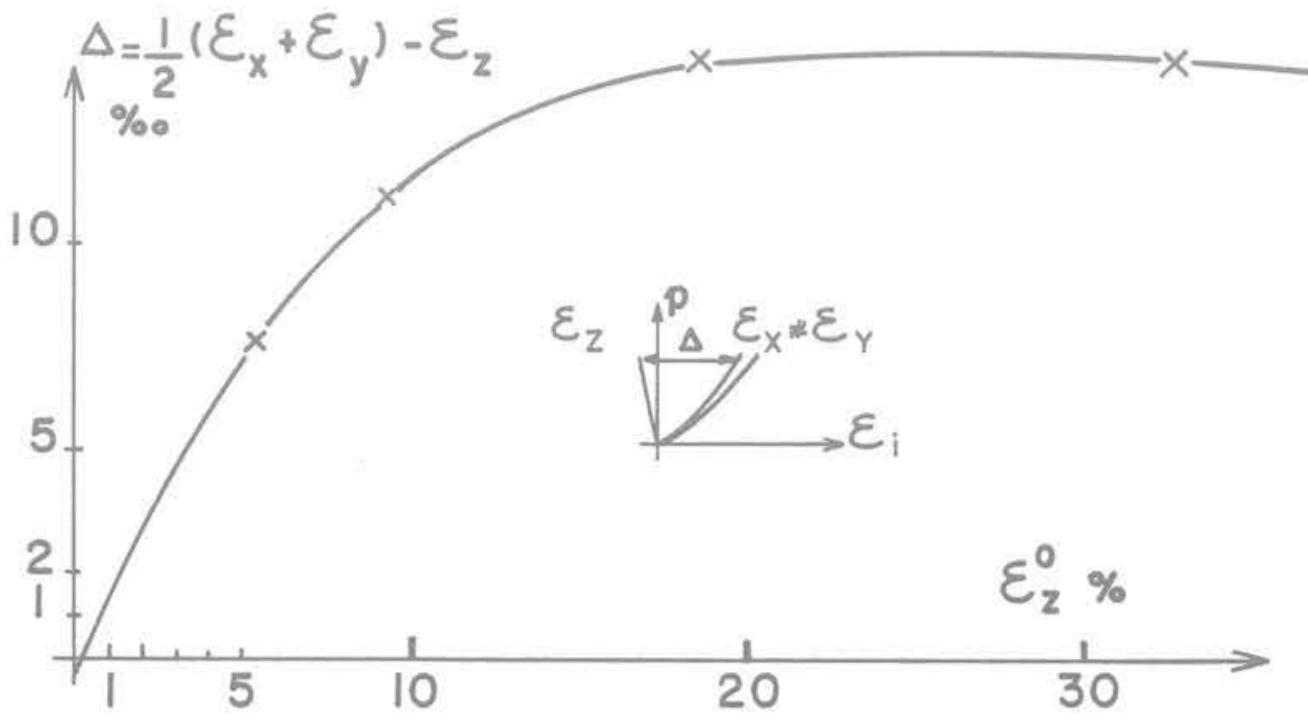

Fig. 4 Evolution de I'orthotropie créée par Tri $\emptyset$ 


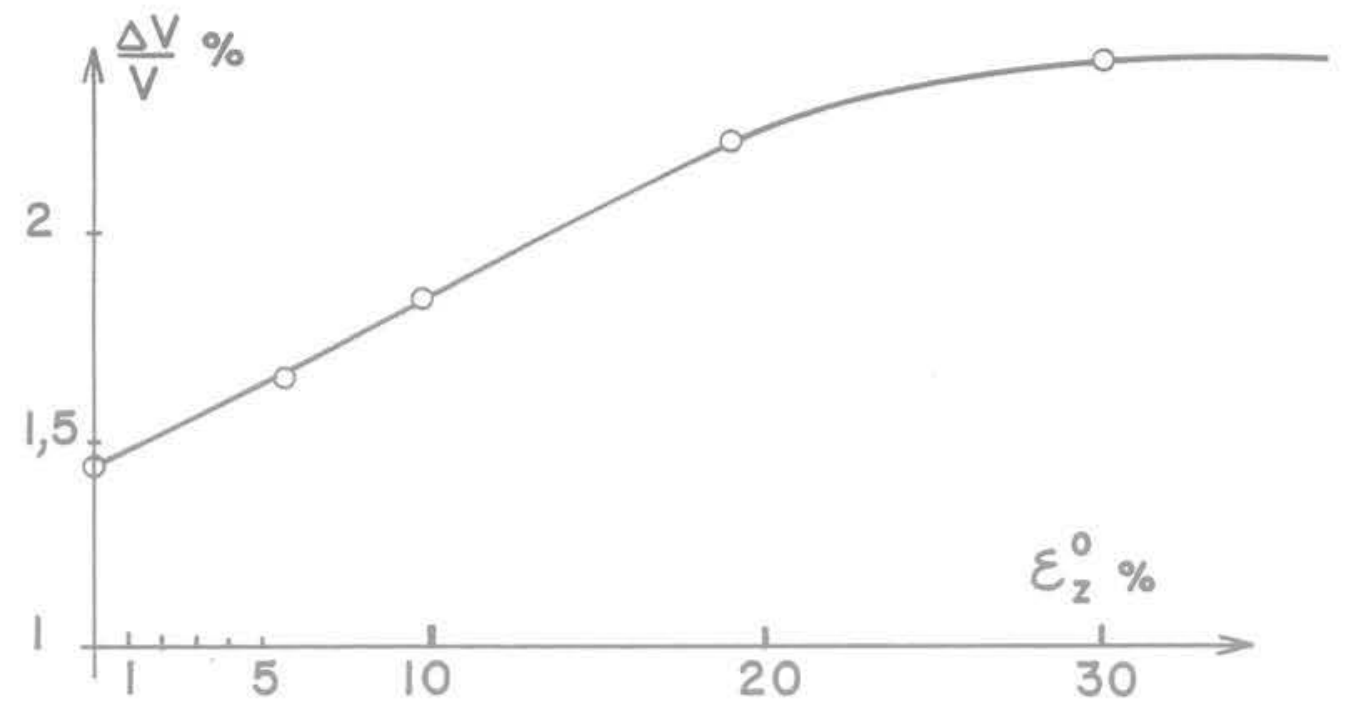

Fig. 5 Evolution du compactage isotrope $(p: 0,3 \rightarrow 2 \mathrm{MPa})$ en fonction de $\epsilon$ (sable 15)

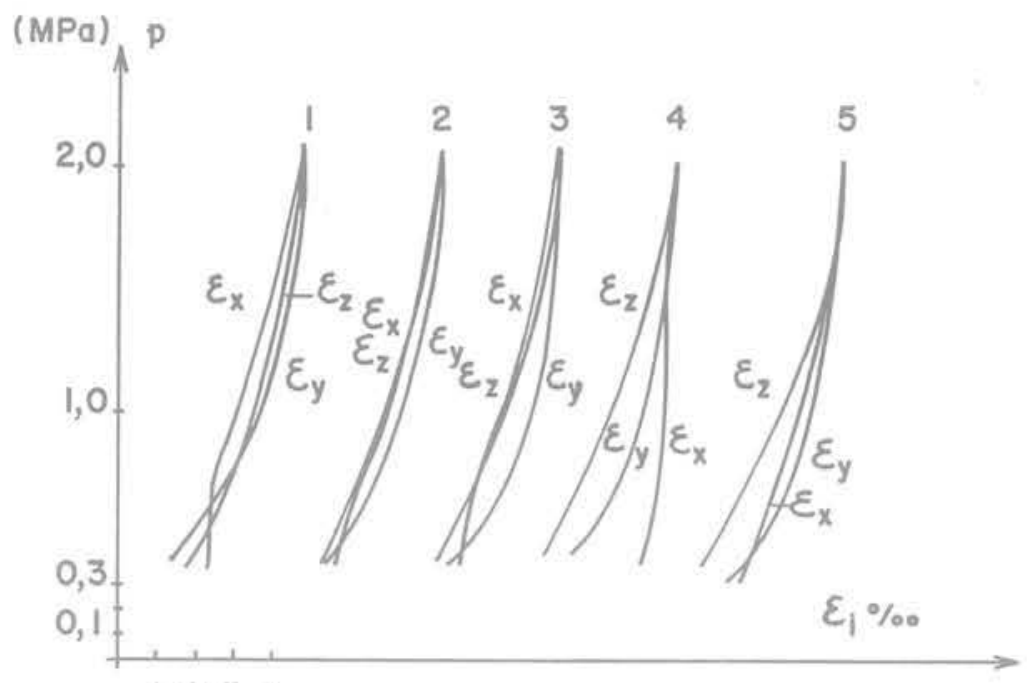

1234

Fig. 6 Courbes de décharge isotrope : $\epsilon_{x} \epsilon_{y} \epsilon_{z}$ ramenés à la même origine. Essais isotropes 1, 2, 3, 4, 5 (sable 15)

(fig. 2) on remarque également que la contrainte est stationnaire.

On peut donc en déduire que l'éçoulement plastique (écoulement à is $=\mathrm{c}^{\text {te }}$ ) ne modifie pas l'anisotropie du matériau. Ou inversement que l'écoulement plastique se produit lorsque l'on a atteint une anisotropie stable.

Observons maintenant les variations de volume. Nous avons représenté sur la figure 5 l'évolution du compactage au cours du chargement isotrope quand $p$ varie de 0.3 a $2.0 \mathrm{MPa}$. On constate que ce pourcentage augmente avec $\varepsilon_{z}^{0}$ et tend également vers une limite.

On attribue généralement cette augmentation du compactage au fait que l'échantillon est de plus en plus lâche au début de chaque essai (cf. fig. 1). Mais il faut noter. à la lumière des résultats précédents, que la mesure de la densité n'est qu'une information globale de l'état de l'échantillon et que, du fait de l'anisotropie, le compactage n'est pas également réparti sur chaque direction.

\subsection{Courbe de décharge isotrope}

Les variations de $z_{x}, z_{\gamma}, z_{z}$ en fonction de $p$ au cours des décharges isotropes sont représentées figure 6 . On remarque que l'anisotropie mise en évidence au cours de la charge n'apparait plus aussi nettement. En particulier $\mathrm{d}_{\mathrm{z}_{z}} / \mathrm{dp}$ est maintenant positif.

Les courbes de recharge, obtenues lors d'autres essais non présentès ici, ont également la même allure. L'anisotropie sur chemin isotrope créée par la cinématique triaxiale de révolution disparaît donc après la première charge. pour les décharges et les recharges isotropes.

On retrouve ici la remarque faite par Elshoby (1973). qui a observé que "la décharge peut être considérée comme isotrope $n$. 

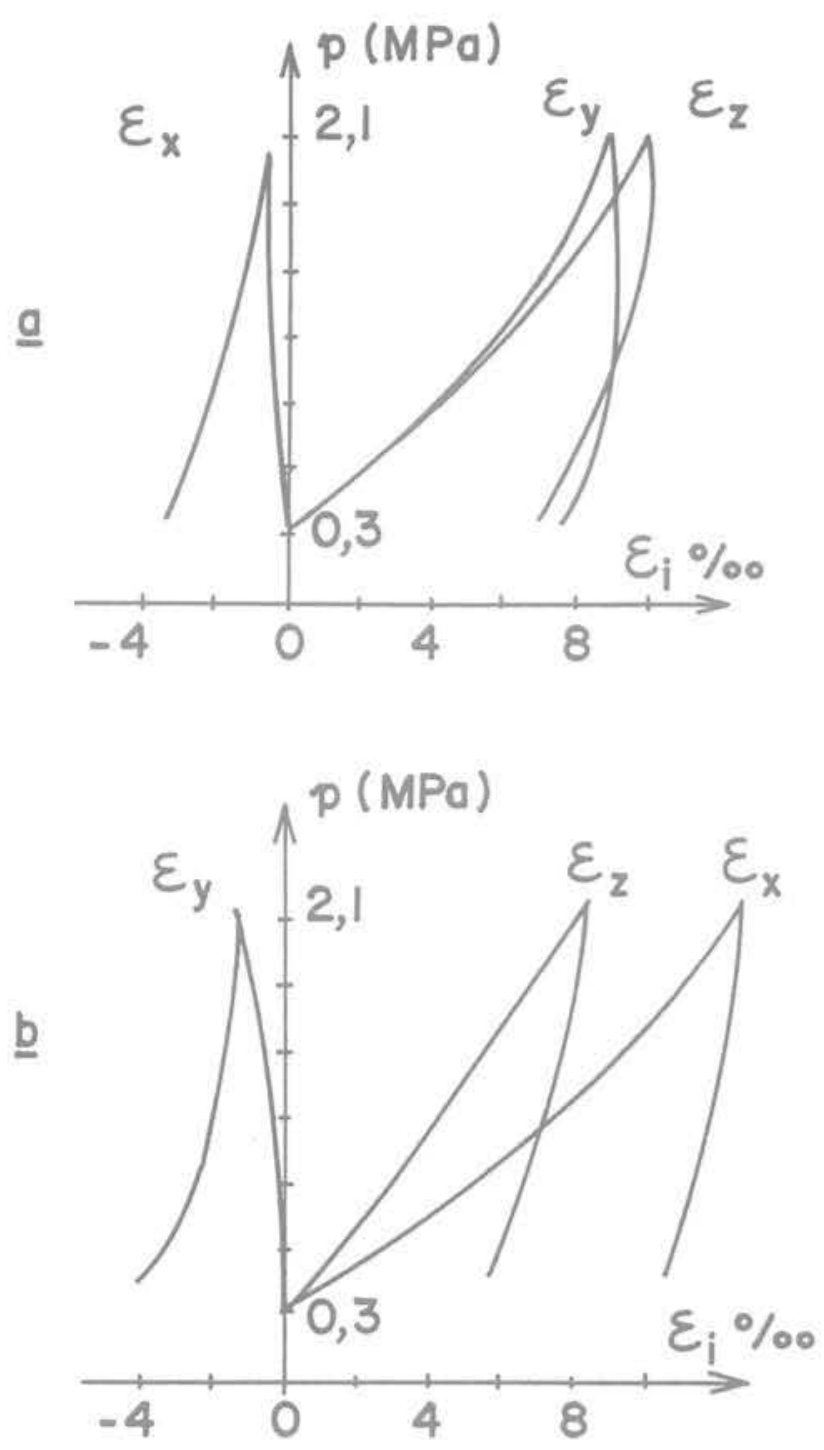

Fig. 7 Cycles isotropes - Variations de $\epsilon_{X} \epsilon_{y} \epsilon_{z}$ en fonction de $p$ (sable 15)
a) après Tri $\emptyset$
b) après Tri ø

\subsection{Cycles isotropes après Tri $\phi_{x}^{\mathrm{C}}$ et Tri $\phi_{Y}^{\mathrm{C}}$}

Pour confirmer ces résultats qui faisaient apparaître que l'anisotropie observée était due à la cinématique triaxiale qui avait précédé l'essai isotrope, nous avons. avec le même échantillon. (fig. 1), réalisé successivement un essai triaxial de révolution dans la direction $\mathrm{X}$. puis dans la direction $Y$. Les résultats, présentés figure 7 , montrent que la réponse du matériau sur chemin isotrope est de nouveau sensiblement orthotrope de révolution et que l'axe d'orthotropie est toujours la direction de compression dans l'essai triaxial de révolution qui a précédé.

\section{Anisotropie créée par les essais triaxiaux d'extension (Sable 17)}

L'essai sable 17 est du même type que l'essai sable 15 présenté précédemment. les sollicitations triaxiales de compression étant remplacées par 2 sollicitations d'extension (noté Tri $\phi_{z}^{E}$ et Tri $\phi_{Y}^{E}$ ) puis une sollicitation de compression (Tri $\phi_{Y}^{\mathrm{C}}$ ).
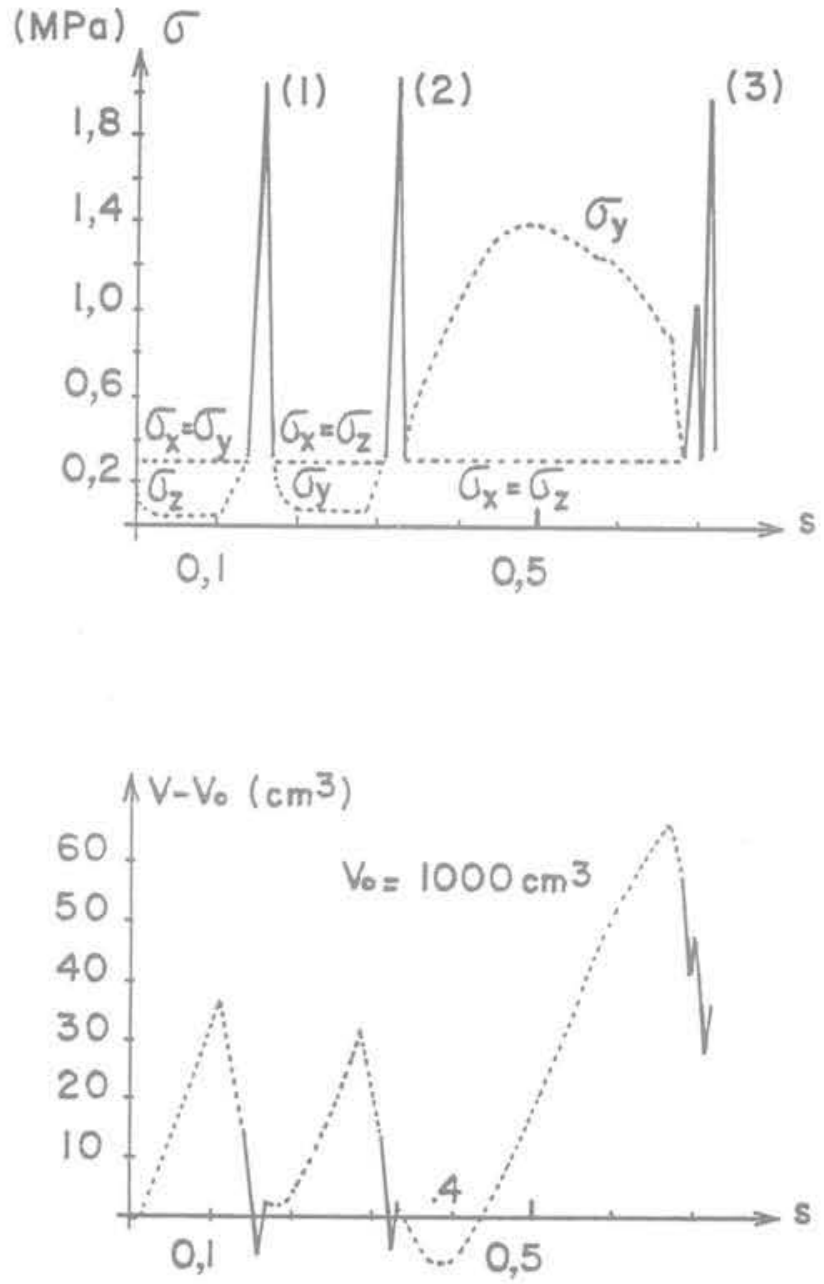

Fig. 8 Evolution des contraintes et du volume (sable 17) essais isotropes essais triaxiaux de révolution

Les variations des contraintes et du volume de l'échantillon sont représentées figure 8 en fonction du paramètre s.

Sur la figure 9 nous avons reporté les variations de $\varepsilon_{\mathrm{X}}$. $F_{r}, r_{z}$ en fonction de $p$ au cours des deux essais isotropes réalisés après les Tri $\phi_{Z}^{\mathrm{E}}$ et Tri $\phi_{y}^{\mathrm{E}}$. On constate encore que la réponse en déformation est orthotrope de révolution par rapport à l'axe de la sollicitation triaxiale qui a précédé l'essai isotrope. Mais maintenant ce sont les deux directions latérales qui se déforment le moins. Or ces directions sont précisément celles qui supportaient les contraintes principales majeures et pour lesquelles $d \varepsilon_{\text {; }}$ était positif (raccourcissement) au cours de la sollicitation triaxiale.

La figure 10 représente les résultats de l'essai isotrope après le Tri $\Phi_{\Upsilon}^{\mathrm{C}}$. On y retrouve les résultats de l'essai (sable 15).

Nous avons de plus réalisé un petit cycle isotrope entre 1 et $0.30 \mathrm{MPa}$. Ce petit cycle est pratiquement isotrope et réversible et confirme la remarque faite en 4.3.

Mais il est très remarquable que la poursuite de la charge de 1 à $2 \mathrm{MPa}$ conserve l'anisotropie observée sur la $1^{\text {re }}$ charge de 0 à $1 \mathrm{MPa}$. D'autres essais seront entrepris pour confirmer ce résultat. 

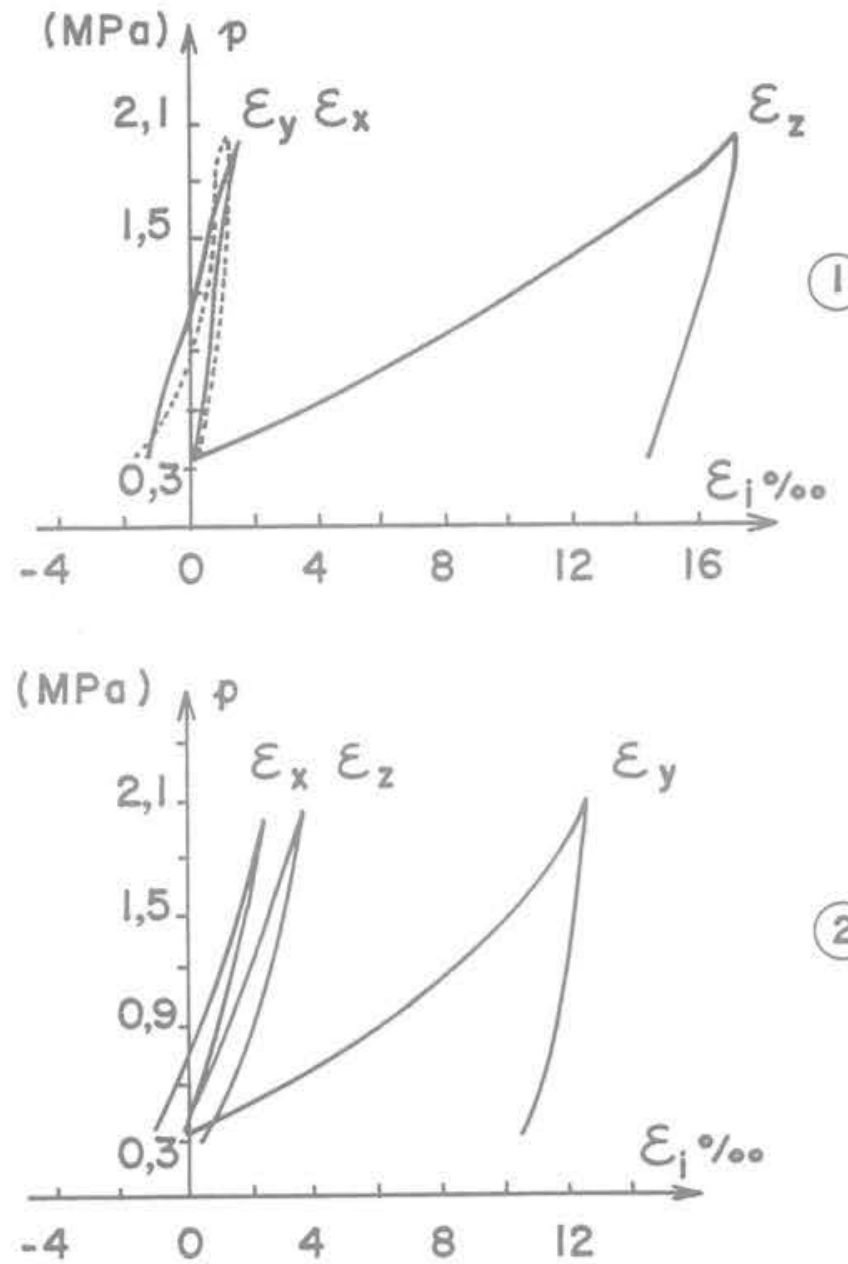

Fig. 9 Essais isotropes $\epsilon_{x} \epsilon_{y} \epsilon_{z}$ en fonction de $p$

1 après Tri $\emptyset$

2 après Tri $\varnothing$

(sable 17)

\section{Anisotropie au cours des essais triaxiaux de révolution}

Les essais isotropes réalisés après les sollicitations triaxiales de révolution ont mis en évidence la structure orthotrope de révolution acquise par le matériau. Que se passe-t-il lorsque l'on sollicite à nouveau l'échantilIon sur un essai triaxial? Deux cas sont à envisager :

\subsection{L'axe triaxial est confondu avec l'axe d'orthotropie de révolution}

C'est le cas des 4 premiers essais, de l'essai sable 15 (fig. 1) oú l'on effectua une $1^{\text {re }}$ charge sur la direction Z et 3 recharges successives, et de l'essai sable 17 (fig. 8) oủ l'on effectua un essai de compression dans la direction $Y$ à la suite un essai d'extension dans la même direction

Nous avons représenté fig. 11 l'évolution des déformations en fonction de la contrainte axiale pour ces différents essais. On note, en comparant les déformations des directions latérales, que la cinématique est proche d'un état de révolution:

\begin{tabular}{|c|c|c|c|c|}
\cline { 2 - 5 } $\begin{array}{c}\text { Sable 15 } \\
\left(\text { Tri } \phi_{Z}^{C}\right)\end{array}$ & $1^{\text {re }}$ charge & $2^{\circ}$ charge & $3^{e}$ charge & $4^{e}$ charge \\
\hline$r_{Z}$ & $5 \%$ & $5 \%$ & $10 \%$ & $16 \%$ \\
\hline$r_{y} / r_{X}$ & 1.15 & 1,25 & 1,21 & 1,10 \\
\hline
\end{tabular}

Sable $17:$ Tri $\phi \frac{E}{Z}\left(1^{r e}\right.$ charge $): f_{z}=-10 \% z_{x} / f_{Y}=1.03$ Tri $\phi_{Y}^{C}$ (après Tri $\phi_{Y}^{E}$ ) $: y_{\gamma}=12 \% r_{x} / \varepsilon_{z}=1,30$.

II faut cependant noter une grande différence de comportement entre les courbes $r_{Z}\left(r_{Z}\right)$ et $\sigma_{Y}\left(r_{\gamma}\right)$. Pour les courbes de recharge 2.3.4 de l'essai sable 15 , le maximum de contrainte est atteint après $2 \%$ de déformation axiale, alors que dans l'essai sable 17 ir n'atteint son maximum quaprés $10 \%$ de déformation. Ces résultats sont tout à fait compatibles avec l'anisotropie. observée: dans le premier cas, nous avions rẻalisé des essais de compression dans la direction $\mathbf{Z}$ qui ont adurci i la direction de compression; dans le deuxième cas, au contraire nous avions réalisé un essai d'extension qui a " amolli " la direction $Y$. Dans les deux cas, les densités initiales étaient respectivement de $\rho=1,65$ pour la courbe $n^{\circ} 4$, et $p=1.73$ pour l'essai Tri $\phi_{r}^{C}$.

Ainsi la différence de comportement entre les 2 courbes ne s'explique pas comme on le fait habituellement en Mécanique des Sols par le fait que l'échantillon est lâche ou dense mais essentiellement par l'anisotropie.

\subsection{L'axe du triaxial n'est pas confondu avec l'axe d'orthotropie de révolution}

C'est le cas des permutations des axes de sollicitations triaxiales

Tri $\phi_{z}^{C}$ puis Tri $\phi_{x}^{C}$ puis Tri $\phi_{r}^{C}$ pour l'échantillon sable 15. Tri $\phi \frac{E}{Z}$ puis Tri $\phi_{Y}^{E} \quad$ pour sable 17

Là figure 12 représente l'évolution des déformations pour ces différents essais. La cinématique n'est plus de révolution : la direction latérale qui n'a pas été axe de révolution se déforme moins que l'autre.

Sable $15:$ Tri $\phi_{x}^{C}$ après Tri $\phi_{Z}^{C}: f_{X}=16 \% q_{Z} / f_{Y}=3,15$

Tri $\phi_{Y}^{\mathrm{C}}$ après Tri $\phi_{X}^{\mathrm{C}}: \varepsilon_{Y}=17 \% \varepsilon_{X} / \varepsilon_{Z}=1,7$

Fig. 10 Essai isotrope $\epsilon_{x} \epsilon_{y} \epsilon_{z}$ en fonction de $p$ après Tri $\varnothing$ (sable 17) 


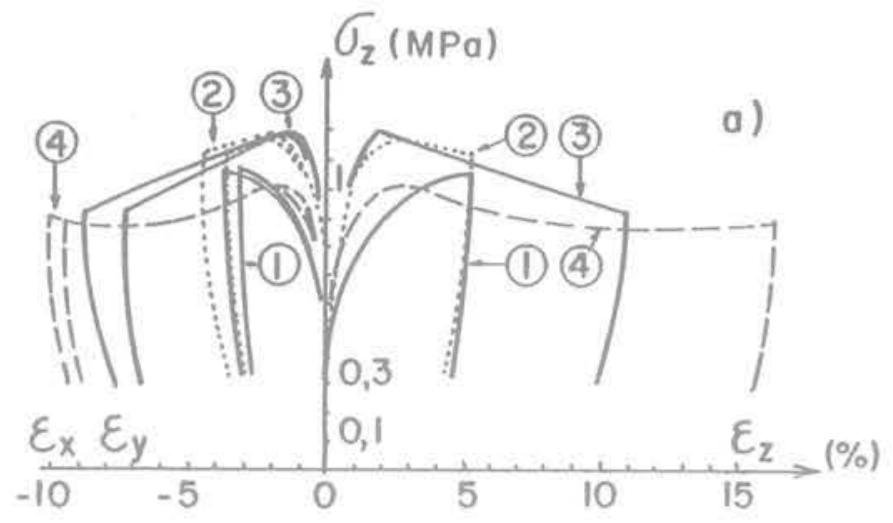

b) $\quad \sigma_{z}(\mathrm{MPa})$

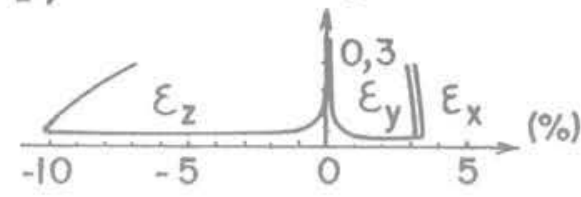

c)

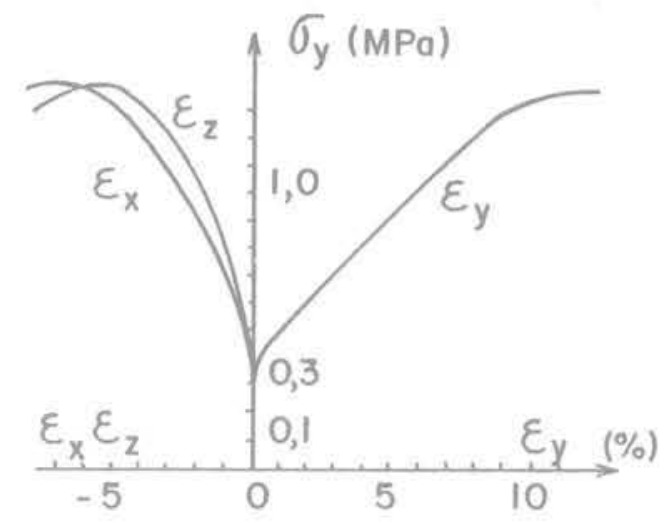

Fig. 11 Evolution des déformations au cours d'essais triaxiaux, l'axe du triaxial étant confondu avec l'axe d'orthotropie de révolution

a) sable 15 - Tri 6

b) sable 17-Tri

c) sable 17-Tri

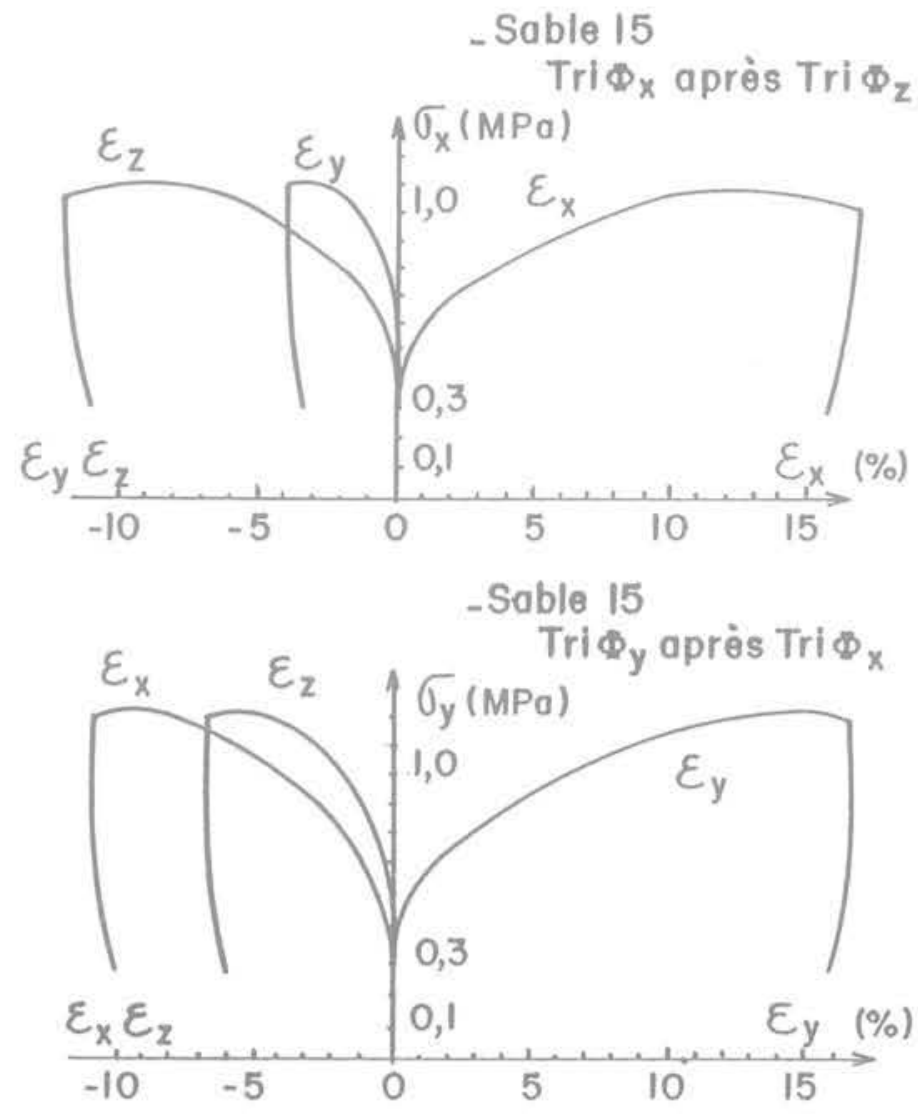

Fig. 12 Evolution des déformations au cours d'essais triaxiaux, l'axe du triaxial n'étant pas confondu initialement avec l'axe d'orthotropie de révolution 
Au cours de ces essais, comme nous l'avons vu grâce aux essais isotropes, il y a modification de l'anisotropie qui évolue d'une orthotropie de révolution selon l'axe du triaxial précédent vers une orthotropie de révolution selon l'axe du triaxial actuel.

Avant de conclure ce chapitre, nous ferons encore une remarque : les essais isotropes nous ont permis de mettre en évidence l'anisotropie de l'échantillon. Nous avons d'autre part indiqué qu'après la $1^{\text {re }}$ charge, les décharges et recharges isotropes étaient sensiblement isotropes aux précisions de nos mesures près. Or nous venons de voir que l'anisotropie ne s'est pas effacée pour les sollicitations triaxiales. On retrouve ainsi une constatation faite par Biarez (1962) : " un échantillon peut paraître isotrope sous pression uniforme et conserver un assemblage anisotrope .

\section{Conclusion}

Les résultats que nous venons de présenter montrent le grand intérêt des presses véritablement tridimensionnelles pour tester le comportement d'un matériau comme le sable.

Rappelons encore notre démarche : nous avons voulu mettre en évidence l'anisotropie créée par une histoire triaxiale de révolution en réalisant, à sa suite, un chemin isotrope en contrainte de façon à nous affranchir de l'anisotropie créée éventuellement par l'existence d'un déviateur de contrainte.

Nous avons mis en évidence les points suivants :

- Dans un essai triaxial de révolution il apparaît une anisotropie qui se stabilise lors de l'écoulement plastique.

- Cette anisotropie n'est pas liée à l'état de contrainte actuel puisqu'elle persiste sur des chemins de contrainte isotrope. II s'agit plutôt d'une anisotropie de structure qui évolue avec la déformation (l'orthotropie selon $Z$ après Tri $\phi_{y}$ devient une orthotropie selon $X$ après Tri $\phi_{\mathrm{x}}$, puis selon $Y$ après Tri $\phi_{\mathrm{r}}$ ).

- Les directions pour lesquelles $d y>0$ (raccourcissement) se udurcissent". Inversement, si $\mathrm{d} \varepsilon>0$ (allongement) on constate un "amollissement ".

- Cette anisotropie s'efface (au moins partiellement) sur les cycles de decharges-recharges isotropes mais réapparaît si l'on poursuit la recharge, ou si l'on réalise un nouvel essai triaxial.
Nous espérons que ces résultats apporteront une meilleure compréhension du comportement des matériaux pulvérulents et par là même, pourront servir de guide dans la formulation d'une loi de comportement.

Notations :

Indice $\mathrm{i}=(\mathrm{X}, \mathrm{Y}, \mathrm{Z})$

$p=\frac{1}{3} \operatorname{tr} \sigma=\frac{1}{3} \sum_{i} \sigma_{i} \quad$ (pression moyenne).

$q=[\operatorname{tr}(\sigma-p \delta)(\sigma-p \delta)]^{1 / 2} \quad$ (intensité du déviateur).

$\varepsilon_{i}=-\log \frac{X_{i}}{X_{i}^{0}} \quad$ (déformation logarithmique).

Tri $\phi_{i}^{C}$, Tri $\phi_{i}^{E}$ essai triaxial de révolution par rapport à l'axe i, de compression ou extension.

$\mathrm{s}=\int_{0}^{t}\left[\left(\operatorname{trd} \varepsilon^{2}\right)\right]^{1 / 2}$

Convention de signe

$\sigma>0$ compression

$\varepsilon>0$ raccourcissement.

\section{Références bibliographiques}

BIAREZ J. (1962) “Contribution à l'étude des propriétés mécaniques des sols et matériaux pulvérulents. " Thèse d'État, Grenoble.

DARVE F., BOULON M. CHAMBON R. (1978) "LOI rhéologique incrémentale des sols. " Journal de Mécanique Vol. 17, $n^{\circ} 5$

ELSHOBY, ANDRAWES (1973) «Experimental examination of sand anisotropy. "Proc 8th ICSMFE, Moscou. 1. pp. 103-109

LANIER J. (1976) *Étude expérimentale des lois de comportement en grandes déformations à l'aide d'une presse réellement tridimensionnelle " Cahier du groupe français de Rhéologie T. IV, 2. 1976

LANIER J. STUTZ P.. COMMANDEUR M. (1979) *Quelques résultats expérimentaux, obtenus à l'aide d'une presse tridimensionnelle, sur les matériaux pulvérulents. J Journée de Rhéologie de I'ENTPE, Lyon. 1979

YAMADA Y. ISHIRAMA K. (1979) «Anisotropic deformation characteristics of sand under threedimensionnal stress conditions." Soils and Foundations. JSSMFE. Vol. 19, 2, 1979 
